\title{
Análise das alterações anatomopatológicas de vísceras bovinas identificadas na inspeção post mortem em um abatedouro-frigorífico de Januária, Minas Gerais
}

Gabriela Silveira Mota ${ }^{1 *}$, Tarcísio Macedo Silva ${ }^{2}$, Luiz Felipe Nunes Menezes Borges ${ }^{3}$, Franciane Gabrielle dos Santos $^{4}$

DOI: https://doi.org/10.35699/2447-6218.2021.35261

\begin{abstract}
Resumo
Objetivou-se analisar as causas e a distribuição das lesões em vísceras bovinas em um abatedouro-frigorífico recém-inaugurado no município de Januária/MG durante o ano de 2018, a partir de relatórios diários de condenação preenchidos durante a inspeção post mortem pelo Serviço de Inspeção Municipal (SIM). Foram abatidos 5.533 bovinos, dos quais 962 animais $(17,3 \%)$ tiveram as vísceras condenadas. O pulmão foi o órgão que apresentou o maior índice de condenações com $66,64 \%$, seguido dos rins $(17,5 \%)$, fígado $(12,7 \%)$, coração $(2,8 \%)$, baço $(0,1 \%)$, e cabeça $(0,1 \%)$. Dentre as causas mais frequentes de condenações por órgão destacaram-se: aspiração de conteúdo ruminal $(37,9 \%)$, cistos (48,5\%), abscessos (80,4\%), pericardite (40,7\%), neoplasia (100\%) e cisticercose calcificada (100\%). Os resultados revelaram lesões de natureza inflamatória, circulatória, parasitária e hemodinâmica, além de falhas no processo de abate.
\end{abstract}

Palavras-chave: Vísceras. Condenações. Inspeção.

\section{Analysis of alteration anatomopathological of bovine vicera identified in post mortem inspection in an industrial slaughterhouse de Januária - MG}

\begin{abstract}
The present study aimed to analyze the causes and distribution of lesions in bovine viscera in an industrial slaughterhouse recently opened in the municipality of Januária / MG during the year 2018, from of daily reports of condemnation completed during post mortem inspection by the municipal inspection service (SIM). 5.533 bovines were slaughtered and being 962 animals (17.3\%) had condemned viscera. The lung was the organ which presented the highest condemnation rate, with $66.6 \%$, followed by the kidneys (17.5\%), liver (12.7\%), heart (2.8\%), spleen (0.1\%), and head $(0.1 \%)$. The most frequent cause of condemnation per organ were: aspiration of ruminal content (37.9\%), cysts (48.5\%), abscesses $(80.4 \%)$, pericarditis (40.7\%), neoplasia (100\%) and calcified cysticercosis (100\%). The results revealed lesions of inflammatory, circulatory, parasitic and hemodynamic nature, as well as failures in the slaughter process.
\end{abstract}

Keywords: Viscera. Condemnation. Inspection.

\footnotetext{
${ }^{1}$ Universidade Federal de Minas Gerais. Instituto de Ciências Agrárias, Montes Claros, MG, Brasil; Serviço de inspeção Municipal de Januária/MG. https://orcid.org/0000-0001-5001-6762

${ }^{2}$ Serviço de Inspeção Municipal - SIM, Januária, Minas Gerais, Brasil. https://orcid.org/0000-0003-2566-8253

${ }^{3}$ Secretaria Municipal de Contagem, Minas Gerais, Brasil. https://orcid.org/0000-0002-7222-7621

${ }^{4}$ Universidade Federal de Minas Gerais. Instituto de Ciências Agrárias, Montes Claros, MG, Brasil

https://orcid.org/0000-0002-7552-3266

*Autor para correspondência: gsmota.vet@gmail.com
}

Recebido para publicação em 15 de Julho de 2021. Aceito para publicação em 30 de Julho de 2021

e-ISSN: 2447-6218 / ISSN: 2447-6218. Atribuição CC BY. 


\section{Introdução}

A bovinocultura de corte configura como um importante segmento do agronegócio, face à expressiva representatividade dessa atividade para a economia brasileira. Dados recentes apontam que em 2017 o rebanho bovino brasileiro chegou a 221,8 milhões de cabeças, sendo abatidos 39,2 milhões de animais. Desse total de animais abatidos $20,9 \%$ foram destinados à exportação e 79,0\% destinados ao mercado interno, totalizando um consumo médio de $37,5 \mathrm{~kg}$ de carne bovina por habitante (ABIEC, 2018).

Os produtos de origem animal são altamente perecíveis e a garantia de qualidade e segurança alimentar desses produtos encontra-se relacionada com a sanidade animal e com o ambiente no qual estão sendo processados. Durante as etapas de inspeção post mortem, realizada pelo serviço de inspeção sanitária oficial, são observadas a existência de alterações macroscópicas patológicas devido às zoonoses e também aquelas que apresentam aspecto repugnante. Neste processo, havendo a presença de alterações e lesões em carcaças e vísceras que as tornem impróprias ao consumo, as mesmas são encaminhadas ao Departamento de Inspeção Final, para as providências cabíveis (Herenda et al., 1994; Brasil, 2017). As lesões determinantes de condenação podem ocorrer devido às questões sanitárias ou falhas de manejo, sejam elas na propriedade de origem, no transporte e/ ou no processo de abate. Sendo assim, o registro dessas lesões garante o auxílio na elaboração de diagnósticos e na adoção de medidas, que incluem a orientação aos produtores, desenvolvimento de políticas no âmbito de saúde pública, além da redução das perdas econômicas nos abatedouros-frigoríficos decorrentes de condenações durante o processo de abate (Lima et al., 2007; Castro e Moreira, 2011; Silva et al., 2013).

Nesse contexto, o presente estudo objetivou analisar as causas e a distribuição das principais alterações anatomopatologias identificadas durante as etapas inspeção post mortem em um abatedouro-frigorífico registrado sob serviço de inspeção municipal recém-inaugurado e localizado no município de Januária, Minas Gerais (MG), no ano de 2018.

\section{Material e métodos}

Foi realizado um estudo epidemiológico descritivo, retrospectivo e secundário a partir de relatórios diários de condenação preenchidos durante a inspeção post mortem dos animais, realizada pelo médico veterinário e/ ou pelos fiscais do Serviço de Inspeção Municipal (SIM) de Januária, durante o período de janeiro a dezembro de 2018. Os dados relacionados às lesões determinantes de condenação foram tabulados usando o programa Excel ${ }^{\circledR}$, separados por órgãos e sistemas e analisados por meio das frequências.
Segundo informações do SIM, esses animais eram provenientes de várias propriedades da região do norte do estado de Minas Gerais, que se caracteriza por utilizar, principalmente, a cria e ciclo completo como sistema de criação (FAEMG \& SENAR, 2016). Como critério de julgamento para as causas de condenações foram considerados condenados os órgãos que apresentaram alterações macroscópicas baseadas nos parâmetros propostos no Regulamento da Inspeção Industrial e Sanitária de Produtos de Origem Animal - RIISPOA (Brasil, 2017).

\section{Resultados e discussão}

Durante o período avaliado foram abatidos 5.533 animais, o que representou uma média de 18 animais por dia. Desse total, 962 animais $(17,3 \%)$ apresentaram algum tipo de lesão em vísceras, fator determinante para a condenação, como pode ser visto na tabela 1 . Os resultados observados corroboram com os descritos por Israel et al. (2014), que também relataram maior acometimento dos pulmões $(36,1 \%)$, seguida dos rins $(29,6 \%)$ e fígado (13.4\%). Percentuais elevados de condenação de pulmões semelhantes aos observados no presente estudo também foram relatados por Almeida et al. (2017), os quais obtiveram $72,0 \%$ de pulmões condenados em um abatedouro municipal localizado no estado de Pernambuco. Já os valores encontrados Silva et al. (2013), em Uberlândia/MG, divergem dos demais, sendo o sistema renal $(66,4 \%)$ o mais acometido por alterações anatomopatológicas passíveis de condenações.

Além de outras alterações, são critérios de julgamento para condenação de pulmões lesões patológicas de origem inflamatória, infecciosa, parasitária, traumática ou pré-agônica (Brasil, 2017). As principais causas de condenações de pulmões, como podem ser observadas na Tabela 2 , indicam falhas na operacionalização durante algumas etapas do abate, em especial no período de descanso, manejo pré-abate, insensibilização e sangria. Daguer (2004) define que causas como aspiração de sangue e a aspiração de conteúdo ruminal são chamadas de tecnopatias, ao passo que são provenientes de lesões operacionais não relacionadas à condição patológica, ou seja, não possuem relação com a sanidade ou estado clínico do animal, sendo descartadas devido ao aspecto repugnante que causam aos olhos do consumidor. Outros achados relacionados às alterações no sistema respiratório foram: a hemorragia, congestão pulmonar, bronquite, edema pulmonar, aderência pleural e abscessos pulmonares. Marino et al. (2016) concluíram que enfisema $(33,0 \%)$, congestão $(10,9 \%)$, aspiração de sangue $(34,6 \%)$ e aspiração de conteúdo ruminal $(21,5 \%)$ foram as cinco principais lesões pulmonares encontradas durante a inspeção post mortem, no Paraná, o que corroborou com os resultados deste trabalho. Almeida et al. (2017) reportaram valores aproximados aos observados neste estudo para causas de hemorragia $(10,9 \%)$, congestão pulmonar $(2,4 \%)$ e edema pulmonar (2,0\%), no entanto, seus achados para abscessos 
Análise das alterações anatomopatológicas de vísceras bovinas identificadas na inspeção post mortem em um abatedouro-frigorífico de Januária, Minas Gerais

pulmonares foram superiores (4,0\%). Da mesma forma, Israel et al. (2014) descreveram um percentual de 6,6\% para bronquite. Diante disso, verifica-se que as lesões relacionadas ao estado clínico dos animais apresentam menor percentual quando comparado aos problemas envolvendo o manejo do abate. Este fato, porém, não deixa de revelar a existência de processos infecciosos que podem comprometer o desempenho produtivo do rebanho.

Tabela 1 - Órgãos condenados durante a inspeção post mortem de bovinos em um abatedouro-frigorífico localizado no município de Januária/Minas Gerais, durante os meses de janeiro a dezembro de 2018

\begin{tabular}{|c|c|c|}
\hline \multirow{2}{*}{ Órgão acometido } & \multicolumn{2}{|c|}{ Frequência Acumulada } \\
\hline & $\mathbf{n}$ & $\%$ \\
\hline Pulmão & 641 & 66,7 \\
\hline Rins & 169 & 17,6 \\
\hline Fígado & 123 & 12,7 \\
\hline Coração & 27 & 2,8 \\
\hline Baço & 01 & 0,1 \\
\hline Cabeça & 01 & 0,1 \\
\hline Total & 962 & 100 \\
\hline
\end{tabular}

n - Frequência Absoluta; \% - Frequência Relativa. Número de bovinos examinados: 5.533.

Tabela 2 - Lesões macroscópicas e/ou causas de condenação em pulmões de bovinos abatidos em um abatedouro-frigorífico localizado no município de Januária/Minas Gerais, durante os meses janeiro a dezembro de 2018

\begin{tabular}{|c|c|c|c|}
\hline \multirow{2}{*}{ Órgão condenado } & \multirow{2}{*}{ Causa e/ou lesão } & \multicolumn{2}{|c|}{ Frequências } \\
\hline & & $\mathbf{n}$ & $\%$ \\
\hline \multirow{9}{*}{ Pulmão } & Aspiração de conteúdo ruminal & 243 & 37,9 \\
\hline & Aspiração de sangue & 165 & 25,8 \\
\hline & Enfisema pulmonar & 78 & 12,1 \\
\hline & Hemorragia (petéquia e equimose) & 70 & 11,0 \\
\hline & Congestão pulmonar & 31 & 4,9 \\
\hline & Bronquite & 22 & 3,4 \\
\hline & Edema pulmonar & 24 & 3,8 \\
\hline & Aderência Pleural & 7 & 1,0 \\
\hline & Abscesso pulmonar & 1 & 0,1 \\
\hline Total & & 641 & 100 \\
\hline
\end{tabular}

n - Frequência Absoluta; \% - Frequência Relativa. Número de bovinos examinados: 5.533.

Em relação as causas relacionadas às condenações de rins (Tabela 3), verificou-se que cistos renais constituem o principal motivo de rejeição e como causa de menor representatividade aparecem as neoplasias renais. Em estudos realizados em Itaiatuba/Pará, Gurgel et al. (2017) também encontraram maior percentual de condenações de rins devido à cistos renais (30,8\%). Por outro lado, e contradizendo os resultados do presente trabalho, em um estudo ocorrido no município de Uberlândia/MG, Silva et al. (2013), reportaram como principais ocorrências de condenações de rins a isquemia $(38,4 \%)$, seguida de congestão $(27,0 \%)$, cisto urinário $(11,0 \%)$ e nefrite $(9,9 \%)$.
Mendes et al. (2009), avaliando morfologicamente rins condenados no oeste e planalto catarinense, encontraram percentuais que se assemelham aos do presente estudo para enfarto renal, com 9,4\%. Como a segunda maior causa de condenação aparece a rejeição por nefrite, a qual apresenta-se normalmente como do tipo intersticial, sendo rara sua apresentação como uma doença clínica em animais pecuários (Radostits et al., 2002). Ressalta-se que, na unidade frigorífica em questão, observou-se que em específico, animais provenientes de determinadas fazendas da região apresentaram uma maior propensão a problemas renais, o que pode indicar manejo nutricio- 
Mota, G. S. et al.

nal inadequado, uma vez que a baixa ingestão de fibra, associado ao baixo consumo de água, bem como elevados níveis de fósforos na ração são considerados fatores para a formação de urólitos que ocasionam os cistos urinários (Loretti et al., 2003).

Tabela 3 - Lesões macroscópicas e/ou causas de condenação de rins bovinos abatidos em um abatedouro - frigorífico localizado no município de Januária/Minas Gerais, durante os meses janeiro a dezembro de 2018

\begin{tabular}{|c|c|c|c|}
\hline \multirow{2}{*}{ Órgão condenado } & \multirow{2}{*}{ Causa e/ou lesão } & \multicolumn{2}{|c|}{ Frequências } \\
\hline & & $\mathbf{n}$ & $\%$ \\
\hline \multirow{8}{*}{ Rins } & Cistos & 82 & 48,6 \\
\hline & Nefrite & 39 & 23,0 \\
\hline & Hidronefrose & 15 & 8,9 \\
\hline & Enfarto Renal & 14 & 8,3 \\
\hline & Icterícia & 7 & 4,2 \\
\hline & Urolitíases & 6 & 3,6 \\
\hline & Pielonefrite & 4 & 2,3 \\
\hline & Neoplasia & 2 & 1,1 \\
\hline Total & & 169 & 100 \\
\hline
\end{tabular}

n - Frequência Absoluta; \% - Frequência Relativa. Número de bovinos examinados: 5.533.

O fígado é considerado um importante órgão vital, devido às funções orgânicas que ele exerce. Em virtude disso, o mesmo torna-se suscetível à determinadas lesões. Considerando as condenações do fígado (Tabela 4), os resultados mostraram ser equivalentes aos observados por Almeida et al. (2017) no estado de Pernambuco, e por Souza et al. (2017), na Zona da Mata mineira, que também constataram uma maior ocorrência de lesões hepáticas por abscessos. Em contrapartida, levantamentos realizados no Triângulo Mineiro e no estado de Santa Catarina reportaram que as lesões hepáticas de maior importância para condenação consistiam em teleangiec- tasia (Mendes e Pilati, 2007; Castro e Moreira, 2011). Essas diferenças podem ser explicadas por diferentes métodos de manejo adotados em determinados tipos de criação (Vechiato et al., 2011). Neste estudo encontrou a hidatidose como a segunda principal causa de condenação de fígado. Este dado torna-se relevante devido ao fato da mesma ser considerada como uma importante zoonose, embora pouco prevalente, e ter sido observada na rotina post mortem do frigorífico. Resultados semelhantes foram encontrados por Brant \& Costa (1963) para o Estado de Minas Gerais, com ocorrência de $6,44 \%$ e na região de Montes Claros/MG, percentual de 5,69.

Tabela 4 - Lesões macroscópicas e/ou causas de condenação de fígado de bovinos abatidos em um abatedouro-frigorífico localizado no município de Januária/Minas Gerais, durante os meses janeiro a dezembro de 2018

\begin{tabular}{|c|c|c|c|}
\hline \multirow{2}{*}{ Órgão condenado } & \multirow{2}{*}{ Causa e/ou lesão } & \multicolumn{2}{|c|}{ Frequência } \\
\hline & & $\mathbf{n}$ & $\%$ \\
\hline \multirow{6}{*}{ Fígado } & Abscesso & 99 & 80,5 \\
\hline & Hidatidose & 8 & 6,5 \\
\hline & Friabilidade & 7 & 5,7 \\
\hline & Neoplasia & 5 & 4,0 \\
\hline & Teleangiectasia & 3 & 2,5 \\
\hline & Icterícia & 1 & 0,8 \\
\hline Total & & 123 & 100 \\
\hline
\end{tabular}

n - Frequência Absoluta; \% - Frequência Relativa. Número de bovinos examinados: 5.533.

Com relação às condenações cardíacas (Tabela 5), o percentual encontrado neste estudo para pericardite se assemelha aos achados descritos por Silva et al. (2013), que relataram 40,8\%. As lesões do pericárdio estão asso- 
Análise das alterações anatomopatológicas de vísceras bovinas identificadas na inspeção post mortem em um abatedouro-frigorífico de Januária, Minas Gerais

ciadas a doenças sistêmicas ou a lesões em outras partes do coração ou tecidos adjacentes, não sendo comum uma afecção isolada do pericárdio (Cotran et al., 1999). Em contrapartida, Gurgel et al. (2017), encontraram como a principal causa de condenação a congestão cardíaca $(51,2 \%)$. No entanto, no presente estudo observou-se que tal condição apresentou menor percentual.

Tabela 5 - Lesões macroscópicas e/ou causas de condenação de coração de bovinos abatidos em um abatedouro-frigorífico localizado no município de em Januária/Minas Gerais, durante os meses janeiro a dezembro de 2018

\begin{tabular}{|c|c|c|c|}
\hline \multirow{2}{*}{ Órgão condenado } & \multirow{2}{*}{ Causa e/ou lesão } & \multicolumn{2}{|c|}{ Frequência } \\
\hline & & $\mathbf{n}$ & $\%$ \\
\hline \multirow{5}{*}{ Coração } & Pericardite & 11 & 40,8 \\
\hline & Aderência do pericárdio & 8 & 29,6 \\
\hline & Miocardite & 6 & 22,2 \\
\hline & Abscessos & 1 & 3,7 \\
\hline & Congestão & 1 & 3,7 \\
\hline Total & & 27 & 100 \\
\hline
\end{tabular}

n - Frequência Absoluta; \% - Frequência Relativa. Número de bovinos examinados: 5.533.

Em relação ao baço, a única condenação se deu devido à neoplasia; tal órgão é considerado como de predileção para metástases, assim como fígado, pulmões e linfonodos regionais (Rosol \& Gröne, 2016).

Para a cabeça foi realizada uma única condenação devido à cisticercose calcificada. Neste caso, foi adotada a medida de condenação somente da cabeça com liberação da carcaça, pois se tratava de cisto único e calcificado, uma vez que não representa riscos à Saúde Pública (Brasil, 2017).

Considerando-se todas as causas de condenação com relação ao total de bovinos abatidos, observou-se que no período avaliado não foram observadas zoonoses passíveis de serem veiculadas pelo consumo de carne bovina. Avelar et al. (2016), Queiroz et al. (2016) e Bennema et al. 2017 destacaram que no Brasil a distribuição espacial e a prevalência de enfermidades (fasciolose, tuberculose, cisticercose) são influenciadas por uma multiplicidade de fatores, tais como: condições climáticas, ambientais, econômicas, socioculturais, higiênico-sanitárias e tipo de criação do rebanho. Os resultados obtidos não atestam a ausência dessas enfermidades na área deste estudo, possivelmente uma avaliação temporal com maior distribuição geoespacial e um maior número de animais possa fornecer maiores informações acerca da distribuição prevalência nesta região.

\section{Conclusão}

Com base nos resultados obtidos, conclui-se que os órgãos acometidos por condições patológicas corresponderam ao pulmão, rins, fígado, coração, baço e cabeça. Os pulmões apresentaram maiores porcentagens de descarte, tendo como principal causa a aspiração de sangue. Nesta circunstância, é possível inferir que a insensibilização realizada de forma correta é de suma importância para a redução de ocorrências de alterações pulmonares. Em relação aos rins, os cistos e a nefrite foram as principais causas de rejeição, estando relacionadas principalmente ao manejo nutricional. No que diz respeito ao fígado, os abscessos se destacaram, correspondendo a mais de $80 \%$ das condenações por órgão. Em relação ao coração, a pericardite e aderência ao pericárdio foram as principais causas de rejeição do órgão na linha de inspeção, podendo ter como origem inflamatória ou traumática. Por fim, o baço e a cabeça obtiveram os menores índices de condenações, sendo rejeitados pela presença de neoplasia e cisticercose calcificada, respectivamente. Diante do exposto, verifica-se que as alterações anatomopatológicas encontradas na indústria são de natureza inflamatória, circulatória, parasitária e hemodinâmica.

\section{Referências}

Associação Brasileira de Indústrias Exportadoras de Carne - ABIEC. Perfil da Pecuária no Brasil - Relatório Anual. 2018. Disponível em: http://www.abiec.com.br/Sumario.aspx.
Almeida, T, J, O.; Silva, S.C,G.; Torres, M.B.A.M.; Franque, M.P. 2017. Lesões macroscópicas e causas de condenação de carcaças e vísceras de bovinos abatidos na microrregião de Garanhuns, Pernambuco, Brasil. Medicina Veterinária (UFRPE), 11:4, 292-300. Doi: https://doi. org/10.26605/medvet-n4-1959. 
Mota, G. S. et al.

Avelar, B. R.; Marcelino, L.C.; Campos, R.F; Santos, A.R.; Martins I.V.F. 2016. Spatial analysis on the risk of bovine cysticercosis occurrence in the state of Espírito Santo, Brazil. Parasite epidemiology and control, 1:2, 116-123. Doi: https://doi.org/10.1016/j.parepi.2016.04.003.

Bennema, S.C.; Molento, M.B.; Scholte, R.G.; Carvalho, O.S.; Pritsch, I. 2017. Modelling the spatial distribution of Fasciola hepatica in bovines using decision tree, logistic regression and GIS query approaches for Brazil. Parasitology, 144:13, 1677-1685. Doi: https://doi.org/10.1017/ S0031182017000786.

Brant, P.C; Costa, A.S. 1963. Distribuição geográfica da Hidatidose, Cisticercose, Euritrematose, Dictiocaulose e Fasciolose bovinas no estado de Minas Gerais. Arquivos da Escola de Veterinaria da Universidade Federal de Minas Gerais., Belo Horizonte, 15, 47-59.

Brasil. Decreto no 9.013, de 29 de março de 2017. Regulamento de Inspeção Industrial e Sanitária de Produtos de Origem Animal. Disponível em: http://www.planalto.gov.br/ccivil_03/_ato2015-2018/2017/ decreto/d9013.htm.

Castro, R.V.; Moreira, M.D. Ocorrências patológicas encontradas de rins e fígados bovinos em matadouro frigorífico do triângulo mineiro. Cadernos de Pós-Graduação da FAZU, v. 1, 2011. Disponível em: http:// www.fazu.br/ojs/index.php/posfazu/article/viewFile/343/249.

Cotran, R.S., Kumar, V.K.; Collins, T. 1999. Pathologic basis of disease. 6. ed. W.B. Saunders. Philadelphia.

Daguer, H. 2004. Inspeção sanitária de pulmão de suínos. A Hora Veterinária, 24:141, 43-46.

Federação da Agricultura e Pecuária de Minas Gerais - FAEMG; Serviço Nacional de Aprendizagem Rural AR-MG - SENAR. 2016. Diagnóstico da Pecuária de Corte Mineira. Disponível em: http://www.faemg.org. br/Content/uploads/publicacoes/arquivos/Xcui1592321508129.pdf.

Gurgel, A.V.L.; Cirne, L.G.A.; Pereira, M.F.; Castro, S.R.S.; Silva, A.S.L.; Neves, K.A.L.; Cabral, I.S.; Carvalho, G.G.P. 2017. Condenações de vísceras bovinas no município de Itaituba - PA. Agroecossistemas, 9:2, 91-101. Doi: http://dx.doi.org/10.18542/ragros.v9i2.5110.

Herenda, D.P.G.; Chambers, A.; Ettriqui, P.; Seneviratna, T.J.P.S. 1994. Manual on meat inspection for developing countries. Food and Agriculture Organization of the United Nations (FAO), Animal Production and Health Division, Rome, Italy. 234-236.
Israel, L.F.S.; Duarte, M.T.; Carrijo, K.F. Principais causas de condenação em bovinos abatidos em um matadouro frigorífico sob inspeção oficial no município de Rio Branco, Acre, Brasil. Enciclopédia biosfera, v. 10, n.19, p. 1549-1562, 2014. Disponível em: https://www.conhecer.org. br/enciclop/2014b/AGRARIAS/principais\%20causas.pdf.

Lima, M.D.F.C.; Suassuna, A.C.D.; Ahid, S.M.M.; Filgueira, K.D. 2007. Análise das alterações anatomopatológicas durante a inspeção post mortem em bovinos no abatedouro frigorífico industrial de Mossoró, Rio Grande do Norte. Ciência Animal, 17:2, 113-116. Disponível em: http:// www.uece.br/cienciaanimal/dmdocuments/Comunicacao2.2007.2.pdf.

Loretti, A.P.; Oliveira, L.O.; Cruz, C.E.F. 2003. Clinical and pathological study of an outbreak of obstructive urolithiasis in feedlot cattle in southern Brazil. Pesquisa Veterinária Brasileira, 23, 61-64. Doi: https:// doi.org/10.1590/S0100-736X2003000200003.

Marino, P.C.; Bonese, G.L.; Filho, L.C.N.; Furlan, D.; Augusto, D.N.; Bogado, A.L.G.; Marcasso, R.A.; Silva, L.C.; Santos, M.D.; Okano, W. 2016. Lesões pulmonares de bovinos encontradas na inspeção post mortem em matadouros frigoríficos no estado do Paraná. Revista Brasileira de Higiene e Sanidade Animal, 10:4, 669-676. Disponível em: https://dialnet.unirioja.es/servlet/articulo? codigo $=5763271$.

Mendes, R.E.; Moreira, F.; Rocha, C.S.; Pilati, C. 2009. Estudo morfológico de rins de bovinos abatidos em frigoríficos industriais sob inspeção estadual no Oeste e no Planalto de Santa Catarina, Brasil. Ciência Animal Brasileira, 10:1, 281-287. Disponível em: https://www.revistas. ufg.br/index.php/vet/article/view/3292.

Mendes, R.E.; Pilati, C. 2007. Estudo morfológico de fígado de bovinos abatidos em frigoríficos industriais sob inspeção estadual no Oeste e no Planalto de Santa Catarina, Brasil. Ciência Rural, 37:6, 1728-1734. Disponível em: https://www.scielo.br/j/cr/a/ GWWKBwMyTW6vSNV7GKYQ4tr/?format=pdf\&lang=pt.

Queiroz, M.R.; Groff, A.C.M.; Silva, N.S.; Grisi-Filho, J.H.H.; Amaku, M.; Dias, R.A.; Telles, E.O.; Heinemann, M.B.; Ferreira Neto, J.S.; Gonçalves, V.S.P.; Ferreira, F. 2016. Epidemiological status of bovine tuberculosis in the state of Rio Grande do Sul, Brazil. Semina: Ciências Agrárias, 37:5, 3647-3658. Disponível em: https://www.redalyc.org/ pdf/4457/445748364022.pdf.

Radostits, O. M.; Gay, C. C.; Blood, D. C.; Hinchcliff, K.W. 2002. Clínica veterinária: um tratado de doenças dos bovinos, ovinos, suínos, caprinos e equinos. 9 ed. Rio de Janeiro, Guanabara Koogan.

Rosol T.J. \& Gröne A. 2016. Endocrine glands, p.269-357. In: Maxie M.G. (Ed.), Jubb, Kennedy, and Palmer's Pathology of Domestic Animals, 3. Elsevier, St. Louis, Missouri. 